\title{
Role of FKBPs in Parkinson's Disease
}

\author{
Souvik Chattopadhaya, Amaravadhi Harikishore and Ho Sup Yoon \\ School of Biological Sciences, Nanyang Technological University \\ Singapore
}

\section{Introduction}

Parkinson's disease (PD) is the second most common neurodegenerative disease among the elderly. While sporadic PD constitutes $99 \%$ of the cases, the remaining $1 \%$ is of genetic origin. The neuropathological hallmarks of $\mathrm{PD}$ are progressive degeneration of dopaminergic (DA) neurons and presence of Lewy neurites and Lewy bodies (LBs) intracytoplasmic proteinaceous inclusions that contain $\alpha$-synuclein (SYN), synphilin-1, components of the ubiquitin proteasomal pathway and parkin (Dawson, 2006). The loss of DA neurons in substantia nigra pars compacta (SNpc) results in decreased signalling in the striatum thereby giving rise to motor defects like resting tremor, bradykinesia, rigidity and posture instability. Besides DA neuronal loss, microglial activation and increased astroglial and lymphocyte infiltration also occur in PD. A role for inflammation in PD has been inferred from the identification of human leukocyte antigen (HLA)-DR positive reactive microglia in the brains of PD patients (McGeer et al., 1988). Additionally, levels of proinflammatory cytokines like IL-6, IL-1 $\beta$, TNF $\alpha$ have been found to be elevated in the blood and cerebrospinal fluid (CSF) of PD patients (Nagatsu \& Sawada, 2005; Dawson, 2006) Although these inflammatory components might serve as useful biomarkers, the aetiology of striatal DA degeneration still remains enigmatic.

In the last decade, identification of mutations in several distinct genes (LRRK2, parkin, PINK1, DJ-1, $\alpha$-synuclein, MAPT, UCHL1 etc) linked to different forms of familial Parkinsonism has imparted a new direction to understanding PD pathogenesis (Tong \& Shen, 2009). The question as to how seemingly divergent genes cause PD still remains unanswered, as there is no common molecular pathway involving these gene products. While parkin, $\alpha$-synuclein (SYN) and ubiquitin C-terminal hydrolase L1 (UCHL1) are functionally associated with the cellular ubiquitin proteasomal system (UPS), DJ-1 and PINK1 protect against oxidative stress and mitochondrial dysfunction. More recently, microarray analysis of SNpc from parkinsonian brain (Mandel et al., 2005) has shown that 68 genes related to protein degradation, signal transduction, dopaminergic transmission, iron transport and glycolysis are downregulated. Prominent among these are the protein chaperone HSC-70, subunits of the UPS and SKP1A, a member of the E3 ubiquitin ligase complex. Therefore, it is most likely that impairment in energy metabolism and/or alterations in UPS are the underlying mechanisms for PD pathogenesis (Eriksen et al., 2005; Mandel et al., 2005).

Current PD treatment regimes can be divided into three categories: symptomatic, protective and restorative. Only symptomatic treatment via the administration of L-dopa and other 
drugs affecting neurological transmission have shown efficacy. However, side effects like dyskinesia, motor fluctuations and neurological complications limit their long-term use (Gold \& Nutt, 2002). The neuroimmunophilins ligands (NILs) are a promising new class of drugs for treatment of PD as well as other neurodegenerative diseases. NILs are derived from the immunosuppressant, FK506 (tacrolimus) and exert their activity not via any cellular mechanism involving the immune system but by binding to a group of proteins termed FK506 binding proteins (FKBPs). When compared to the immune system, FKBP expression levels are highly enriched (10-50 fold greater) in both the central and peripheral nervous system. In this chapter, we review our current understanding of the role of FKBPs in the nervous system with an emphasis on the protein partners that interface with FKBPs inside cells. For brevity, we limit our discussions to FKBPs that are enriched in the nervous systems and may have important role in Parkinson's disease pathogenesis. We also highlight the mode of action of the NILs with the hope that knowledge of such interaction will enable rationale design of new drugs with improved efficacy for treatment of Parkinson's disease as well as other neurodegenerative disorders.

\section{Role of FKBPs in the nervous system}

FKBPs together with cyclophilins (CyPs) comprise a family of phylogenetically conserved immunophilins that have peptidyl prolyl isomerase activity (PPIase; EC 5.2.1.8), producing the cis-trans isomerization of X-Pro peptide bond, an essential but rate-limiting step in the protein folding process (Barik, 2006). Initial isolation and purification of immunophilins were based on their differential affinity towards the principal immunosuppressant drugs rapamycin, FK506 and cyclosporin A (CsA). While CyPs bind to only CsA, FKBPs have affinities for both FK506 and rapamycin. Immunosuppressive activity mediated by these drugs is brought about by their binding to the cognate immunophilins. The FK506/FKBP or $\mathrm{CsA} / \mathrm{CyP}$ binary complexes bind to the $\mathrm{Ca}^{2+} /$ calmodulin dependent protein serine/threonine phosphatase, calcineurin $(\mathrm{CaN})$ and inhibit its phosphatase activity. The resulting FKBP-FK506-CaN ternary complex cannot dephosphorylate the key transcription factor, nuclear factor of activated T-cells (NF-AT). Inactive NF-AT remains in the cytoplasm thereby preventing interleukin-2 (IL-2) secretion (Figure 1). Consequently, both T-cell activation and proliferation is inhibited. On the other hand, FKBP-rapamycin complex exerts immunosuppression by inhibiting the serine/threonine kinase activity of mammalian target of rapamycin (mTOR) (Sharma et al., 1994).

The role of FKBPs in the nervous system was initiated by observations that the brain is abundantly enriched in CyPs and FKBPs (Maki et al., 1990; Steiner et al., 1992; Dawson et al., 1994). The importance of immunophilins in the nervous system was firmly established from studies showing that FK506 potently (as low as $0.1 \mathrm{nM}$ ) increases neurite outgrowth in both PC-12 (Lyons et al., 1994) and SH-SY5Y cell culture models as well as in primary cultures of chick dorsal root ganglion and hippocampal neurons (Hamilton and Steiner, 1998). Efforts to explain this neurotrophic effect focussed on the calcineurin-dependent pathway involving the CaN substrate, GAP-43 (growth-associated protein-43). GAP-43 selectively localizes to developing neurons and its phosphorylation is known to enhance its neurite extension activity (Meiri et al., 1991). Though initially tenable, the hypothesis was challenged when it was shown that both CsA (Gold, 1997) and non-immunosuppressive (hence non-calcineurin binding) derivatives of FK506 exhibit neurotrophic effect with similar potencies as that of FK506. Therefore, it is most likely that nerve growth proceeds via a calcineurin-independent pathway. 


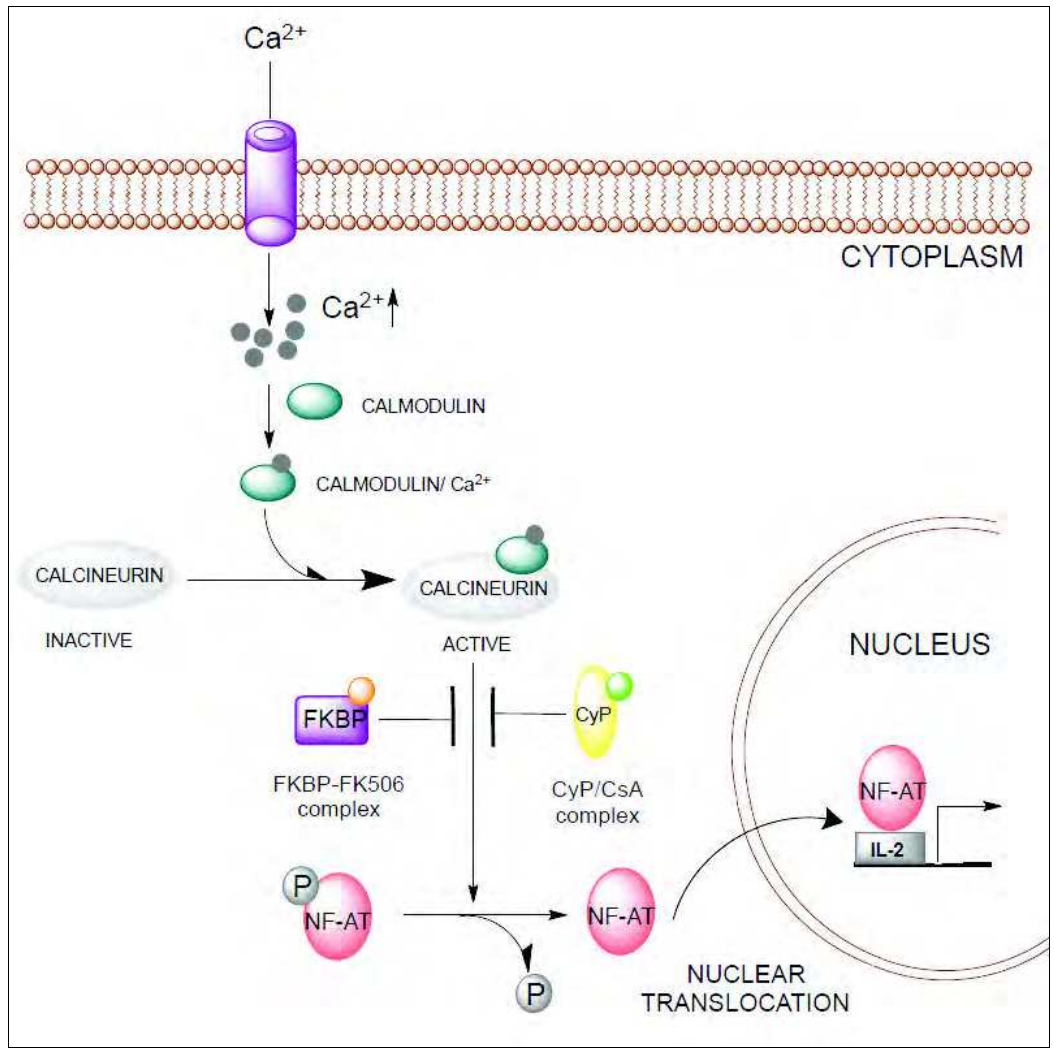

Fig. 1. Immunosuppressive effect of neuroimmunophilin ligands: T-cell receptor activation leads to a rapid increase in intracellular calcium levels with concomitant activation of the $\mathrm{Ca}^{2+} /$ calmodulin-dependent phosphatase, calcineurin $(\mathrm{CaN})$. Active CaN dephosphorylates the transcription factor NF-AT, allowing its nuclear translocation and thereby upregulating IL-2 expression. Addition of FK506 or CsA results in formation of FKBP-FK506-CaN/CyPCsA-CaN ternary complexes that inhibits CaN-dependent NF-AT dephosphorylation, as a result T-cell activation and IL-2 secretion does not occur.

When FK506-treated brain lysates were probed for proteins with increased phosphorylation levels, one of the identified targets was neuronal nitric oxide synthase (nNOS). In the brain, nNOS catalyzes the formation of nitric oxide (NO) from arginine and its catalytic activity is inhibited by phosphorylation. Following cerebral vascular occlusion, there is a massive increase in the excitatory neurotransmitter glutamate. Elevated glutamate levels, acting through the N-methyl-D-aspartate (NMDA) receptor, activates nNOS resulting in increased $\mathrm{NO}$ formation and neurotoxicity (Figure 2). Toxicity may involve NO itself or its combination with superoxide free radical $\left(\mathrm{O}_{2}^{-}\right)$to form peroxy-nitrite that decomposes to hydroxide $\left(\mathrm{OH}^{-}\right)$and $\mathrm{NO}_{2}\left(\mathrm{NO}_{2}^{-}\right)$free radicals with subsequent cellular damage by oxidation of nucleic acids, proteins and membrane lipids (Snyder, 1992). By enhancing phospho nNOS levels, FKBPs inhibit NO formation and thereby attenuate glutamate toxicity following vascular stroke (Snyder et al., 1998). Contrary to nerve regeneration, FKBP- 
mediated neuroprotection proceeds via calcineurin inhibition as anti-stroke effects were also seen with CsA.

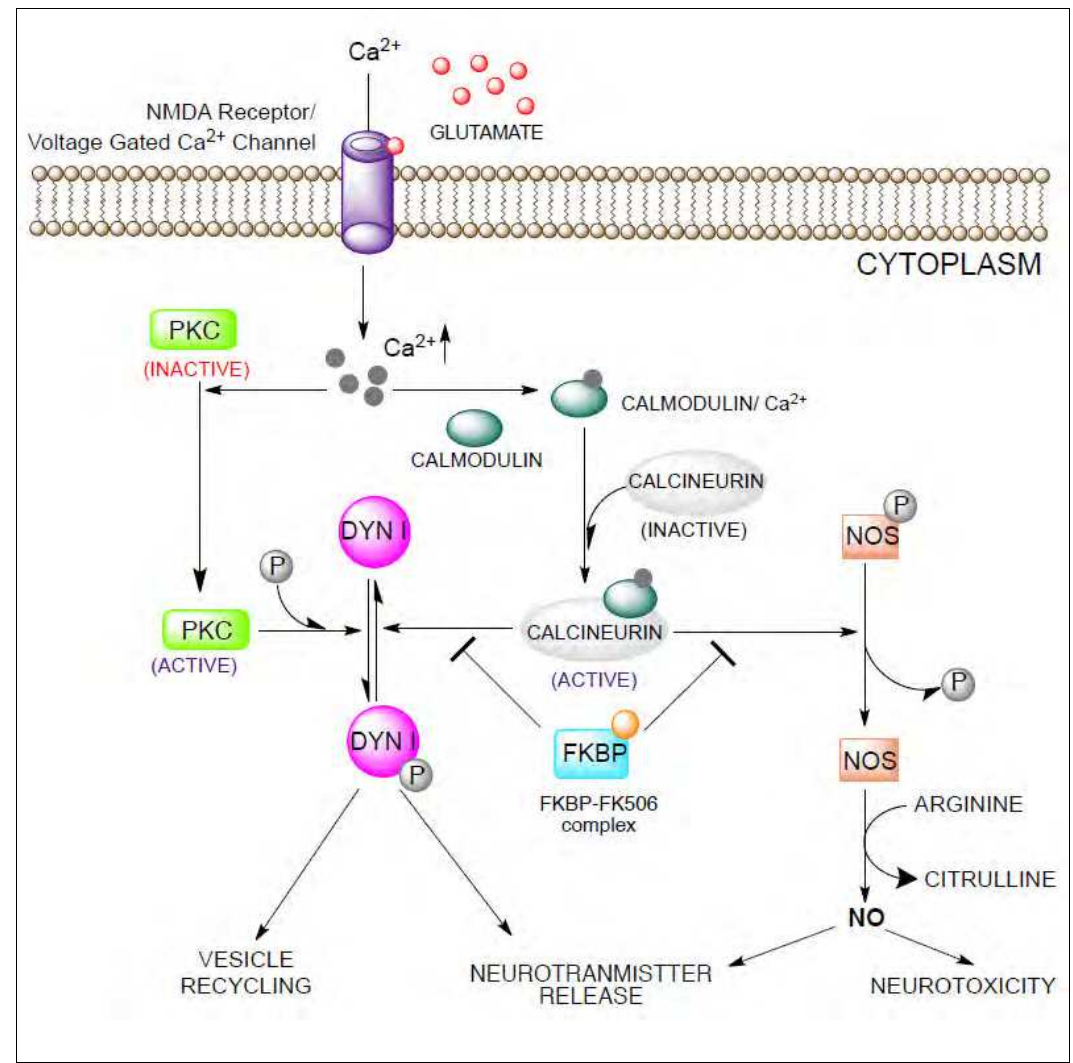

Fig. 2. NILs regulate neurotoxicity and neurotransmitter release: Glutamate-mediated influx of calcium through the NMDA receptor activates calcineurin $(\mathrm{CaN})$ that in turn dephosphorylates $\mathrm{nNOS}$ and increases its catalytic activity. nNOS activation leads to increased $\mathrm{NO}$ formation and subsequent neurotoxicity and neurotransmitter release. FK506/CsA can counteract this neuronal toxicity by inhibiting CaN-dependent nNOS activation. Influx of $\mathrm{Ca} 2+$ also activates $\mathrm{PKC}$ and $\mathrm{CaN}$ that have opposing effect on phosphorylation state of the GTPase, dynamin I. While PKC-mediated phosphorylation of dynamin I increases its GTPase activity and leads to increased neurotransmitter release, $\mathrm{CaN}$ dephosphorylates and inactivates dynamin I. By inhibiting CaN, FK506 and CsA enhances phospho-dynamin I levels and subsequent depolarization-evoked neurotransmitter release.

FK506 has contradictory roles in neurotransmitter release - it inhibits NMDA induced neurotransmitter release while augmenting depolarization-induced release. Since neurotransmitter release proceeds via calcineurin-dependent pathway, this discrepancy in FK506 response can be attributed to the involvement of distinct calcineurin substrates, nNOS and dynamin I (Figure 2). FK506 reduces glutamate release from NMDA-stimulated 
striatal synaptosomes as well as acetylcholine and dopamine release from PC12 cells that have been differentiated by NGF (Steiner et al., 1996). Similar reductions seen with the nNOS inhibitor, nitro-L-arginine, indicates that NO regulates neurotransmitter release in PC12 and synaptosomes. In presence of FK506 and CsA, inhibition of calcineurin and subsequent reduction of nNOS activity results in decreased $\mathrm{NO}$ levels and therefore reduced neurotransmitter release.

In contrast, FK506 fails to inhibit potassium depolarization-evoked neurotransmitter release. Both CsA and L-683590 (FK506 analog that inhibits calcineurin), augment glutamate release from synaptosomes that have been treated with the $\mathrm{K}^{+}$-channel blocker, 4-aminopyridine. In this case, the bona fide calcineurin substrate, dynamin I and not nNOS is involved (Nichols et al., 1994). Dynamin I, a GTPase that regulates vesicular recycling, is active in its phosphorylated form; enhanced GTPase activity results in greater synaptic vesicular trafficking and increased rate of neurotransmitter release. CsA and FK506 mediated inhibition of calcineurin enhances dynamin I phosphorylation and hence its activity.

FK506 and its derivatives have also shown neuroprotective activity in neuropathy models mimicking stroke and dementia. For example, FK506-mediated calcineurin inhibition protects against ischemic brain injury (Sharkey \& Butcher, 1994), desensitizes NMDA receptors (Tong et al., 1995), prevents long-term depression (LTD) in rat hippocampus (Hodgkiss \& Kelly, 1995) and modulates long-term potentiation (LTP) in rat visual cortex (Funauchi et al., 1994). Stabilization of mitochondrial function was suggested to account for the anti-ischemic activities of FK506. FK506 does not target the mitochondrial potential transition pore (MTP) but prevents deterioration in mitochondrial respiration while maintaining cellular ATP levels and $\mathrm{Ca}^{2+}$ homeostasis. Furthermore, the role of FKBPs in the central nervous system has been extensively probed using 1-methyl-4-phenyl-1,2,3,6tetrahydropyridine (MPTP) or 6-hydroxydopamine (6-OHDA) induced lesions of dopaminergic neurons. Both agents cause massive degeneration of nigrostriatal DA neurons thereby making MPTP and 6-OHDA treatment experimental paradigms for Parkinson's disease (Gerlach et al., 1991). Treatment with GPI-1046, a FK506 analog was able to substantially recover the MPTP-damaged DA neurons as evidenced by increased tyrosine hydroxylase staining while rats lesioned with 6-OHDA showed morphological and functional recovery with increased striatal catecholamine levels and reduced amphetamineinduced rotations (Steiner et al., 1997).

How might immunophilin ligands exert their neurotrophic and neuroprotective actions? The data suggests that PPIase activity of FKBPs maybe involved but no conclusive evidence has been provided so far. Several studies have tried to identify the $\mathrm{FKBP}(\mathrm{s})$ involved and elucidate the mechanism-of-action. This has proved particularly difficult as (1) most of the FKBP family members bind FK506 or its derivatives, albeit with varying degrees of affinity; (2) activities of both FKBPs and the protein phosphatase, calcineurin are inhibited by FK506 and its analogues and (3) FKBPs perform multifarious roles in protein folding, translocation and regulation as well as have a wide range of tissue/organellar distribution. Initial studies implicated a role for FKBP12 as elevated mRNA and proteins levels were observed in 6-OHDA model of PD (Nilsson et al., 2007). Moreover, higher levels of FKBP12 have also been reported in brains of PD patients (Avramut \& Achim, 2002). Therefore, a possible link between FKBPs and PD might stem from the increase in expression or redistribution of chaperone proteins in stress conditions. In addition to FKBP12, the human brain is enriched in FKBP38, FKBP51, FKBP52 and FKBP65 (Charters et al., 1994; Coss et al., 1998; Chambraud et al., 2010; Jinwal 
et al., 2010). Collectively, these immunophilins enriched in the nervous system are termed neuroimmunophilins. In the following sections, we will discuss the role of only those neuroimmunophilins that may be important for PD pathogenesis.

\section{Molecular interacting partners of neuroimmunophilins}

The neuroprotective and neurotrophic functions observed with NILs together with studies showing that chaperone proteins like HSP70 can suppresses $\alpha$-synuclein $(\alpha-S Y N)$ mediated loss of dopaminergic neurons in Drosophila model of PD (Auluck et al., 2002; Muchowski, 2002), posits that FKBPs may have important role(s) in preventing PD-associated neurodegeneration. FKBPs, together with other chaperones may convert the toxic conformations of misfolded proteins to non-toxic form that is tolerated by cells. Alternatively, they may prevent the formation of toxic pre-fibrillar intermediates, or accelerate their conversion to nontoxic, amorphous aggregates that can be degraded by the cellular proteolytic machinery.

\subsection{FKBPs interact with $\alpha$-synuclein both in vitro and in vivo}

$\alpha-S Y N$ is a small (140 amino acid) intrinsically disordered protein predominantly localized to the presynaptic terminals. $\alpha$-SYN regulates the functions of several other proteins synphilin-1, parkin, tyrosine hydroxylase, dopamine transporter and phospholipase D, via stoichiometric protein-protein and protein-lipid interactions (Goedert, 2001; Ischiropoulos, 2003). In PD, $\alpha$-SYN aggregates into characteristic fibrillar $\beta$-pleated structures in Lewy bodies. Besides LBs, $\alpha-S Y N$ also forms intermediate-state oligomers that when released from the neurons activate microglia leading to an increased production of ROS and proinflammatory cytokines (Glass et al., 2010). Activated microglia further amplifies this inflammatory response in a positive feedback loop. In $\alpha-S Y N$, the ability of the central hydrophobic NAC (non A $\beta$-component of amyloid plaques in Alzheimer's disease) domain to aggregate is normally counteracted by the highly charged hydrophilic C-terminal domain. Interestingly, all 5 proline residues (Pro ${ }^{108}$, Pro ${ }^{117}$, Pro ${ }^{120}, \mathrm{Pro}^{128}$, Pro ${ }^{138}$ ) of $\alpha$-SYN are located at the C-terminal of the protein. Changes to the C-terminal domain through deletion, point mutations or via posttranslational modifications such as phosphorylation (Kragh et al., 2009) expose the NAC domain leading to hydrophobic interaction driven aggregation. For example, the E3-ubiquitin ligase, parkin can protect against $\alpha$-SYN-induced toxicity by altering the phosphorylation levels of $\alpha$-SYN (Figure 3). By simultaneously reducing PLK2 levels and activating PP2A, parkin decreases Ser ${ }^{87}$ and Ser ${ }^{129}$ phosphorylation thereby decreasing aggregation of phosphorylated $\alpha-S Y N$ in LBs (Khandelwal et al., 2010). The role of FKBPs in synucleinopathy has been probed both in vitro (Gerard et al., 2006) and in vivo (Gerard et al., 2010). In vitro, fluorescence correlation spectroscopy (FCS) measurements showed that addition of FKBP12 accelerates $\alpha$-SYN aggregation into fibrillar structures that mimic aggregates formed in LBs. FKBP12 significantly alters the rate for both the nucleation and fibril formation stages (Gerard et al., 2008). Since FKBPs catalyze the cis-trans isomerization of X-Pro peptide bond, the importance of C-terminal proline residues of $\alpha$ SYN was also investigated. Changing one or more proline residues to alanine increased the aggregation kinetics of mutant $\alpha-S Y N$ (Meuvis et al., 2010). Additionally, FKBP12 did not interact with a proline deficient $\alpha-S Y N$ mutant and this mutant was also found to be more structured. 
Using a neuronal model of synucleinopathy in which a-SYN aggregation and cell death was induced by oxidative stress, Gerard and coworkers have shown that both FK506 and knockdown of FKBP12/FKBP52 can counter the effects of oxidative stress. Likewise, it was shown that overexpression of FKBP12 and -52 enhances a-SYN aggregation. FKBP52 was less potent than FKBP12 in inducing fibrillar aggregation. In vivo, FKBP12 was shown to colocalize with $\alpha-S Y N$ in the brain of A30P- $\alpha-S Y N$ transgenic mice model. Furthermore, FK506 administration reduced both the $\alpha$-SYN aggregation in cells as well as increased survival of $\alpha-S Y N$ overexpressing neurons in the striatum. Collectively, these studies validate FKBPs as a novel target for PD.

Besides $\alpha$-SYN, other interacting partners of FKBP12 identified from 6-OHDA treated rat model of PD include 1-cys peroxyredoxin, HSP70, 14-3-3 zeta, M2-type pyruvate kinase (PKM2), annexin A2 and $\alpha$-enolase (ENO1) (Nilsson et al., 2007). It has been known that levels of PKM2, 14-3-3 zeta and ENO1 are altered during neurodegenerative diseases like PD and Alzheimer's (Poon et al., 2006).

Given that FKBPs are chaperone proteins having important roles in protein folding, it is counterintuitive to note that the interaction between FKBP(s) and $\alpha-S Y N$ results in $\alpha-S Y N$ aggregation. Its is likely that $\alpha-S Y N$ inclusions may not result simply from precipitated misfolded protein but rather from an active process meant to sequester soluble misfolded proteins from the cellular milieu (Kopito, 2000). Accordingly, inclusion body formation might serve as a cellular defense mechanism aimed at removing toxic insoluble proteins.

\subsection{FKBP52 interacts with RET51 in a phosphorylation-dependent manner}

RET51, a tyrosine kinase (TK) receptor, has important roles in the development and maintenance of the nervous system. Recently, FKBP52 was found to be novel interacting partner for RET51 in a split ubiquitin two-hybrid screen (Fusco et al., 2010). Neurotrophins like NGF and glial-cell line derived neurotrophic factor (GDNF) promote the phosphorylation driven formation of RET51/FKBP52 complex; phosphorylation occurs on $\mathrm{Tyr}^{905}$ within the TK domain of RET51 and is a pre-requisite for complex formation. Association of RET51 with FKBP52 does not depend on HSP90 or other chaperones. The involvement of RET51 in PD comes from the genetic analysis of an early onset-PD patient heterozygous for mutations on both RET51 and FKBP52 genes (Fusco et al., 2010). Mutations on both proteins disrupt formation of RET51/FKBP52 complex and its downstream signaling pathway. The detail of this signaling mechanism remains to be elucidated.

\subsection{FKBP38 promotes trafficking of membrane channels}

FKBP38 is distributed to both the mitochondria (Shirane \& Nakayama, 2003) and endoplasmic reticulum (Wang et al., 2005). Only the C-terminal tail is membrane anchored while the bulk of the protein is exposed to the cytosol. The unique topology of FKBP38 allows it to juxtapose between cytosolic and ER chaperone proteins. FKBP38 functions as a co-chaperone to HSC70/HSP90 complex to mediate proper folding and trafficking of membrane proteins like the multidomain cAMP-regulated chloride channel, CFTR (Wang et al., 2006) and the voltage-dependent $\mathrm{K}^{+}$channel, HERG (Walker et al., 2007). The immature form of HERG localizes to the endoplasmic reticulum whereas the fully glycosylated mature protein is present in the Golgi or the cell surface. While siRNA mediated knockdown of FKBP38 reduced HERG maturation, overexpression of FKBP38 was able to rescue the HERG F805C trafficking mutant. FKBP38 is involved in the late stages of HERG folding and ER 
export, as majority of FKBP38 has been found to associate with immature HERG. It is likely that natively folded HERG is released from its final chaperone complex while still attached with FKBP38. The bound FKBP38 could further mediate the attachment of HERG with the motor protein kinesin for transport to the plasma membrane. HERG mutations have been associated with the Long QT syndrome, a cardiac disorder characterized by long QT intervals. PD patients have an increased susceptibility to cardiac arrest as is evident from a prolongation of the QT interval (Hurst et al., 2003). Therefore, it is likely that FKBP38mediated HERG trafficking plays an important role in PD pathogenesis.

\subsection{FKBP mediated regulation of Tau function and its effect on microtubule dynamics}

Tau, a member of the microtubule-associated protein family (MAP), binds and stabilizes microtubules (MTs) and is therefore intrinsically linked with MT dynamics. Six isoforms of tau are present in humans, the longest one having four MT-binding repeat motifs. Normal biological functions of tau are dependent on its phosphorylation state. Involvement of tau in PD pathogenesis comes from the observations that (1) it accumulates in LBs together with $\alpha$ SYN (Ishizawa et al., 2003); (2) analysis of synapse-enriched fractions from PD brains show an increased phosphorylation for both tau and $\alpha$-SYN (Muntane et al., 2008) and (3) tau and synuclein synergistically promote in vitro fibrillization of each other (Giasson et al., 2003). $\alpha$ SYN mediates tau phosphorylation at Ser $262 / 356$ by activating protein kinase A (PKA)(Jensen et al., 1999) while in MPTP models of PD, $\alpha-S Y N$ recruits GSK $\beta 3$ kinase to phosphorylate tau at Ser ${ }^{396 / 404}$ (Duka et al., 2009). Hyperphosphorylation of tau results in MT destabilization by interfering with its binding.

Recent studies have shown that both FKBP51 (Jinwal et al., 2010) and FKBP52 (Chambraud et al., 2007; Chambraud et al., 2010) can interact with tau. FKBP52 preferentially binds to hyperphosphorylated tau and colocalizes with tau at the growth cones in both cortical neurons and PC12 cells. Interestingly, FKBP52 could inhibit tau-mediated tubulin polymerization in vitro. This is consistent with the observation that overexpression of FKBP52 impairs neurite outgrowth in cultured neurons (Chambraud et al., 2010). Interaction of FKBP52 with tau was mapped to the C-terminal TPR domain of FKBP52 (Chambraud et al., 2007). Currently it is not known if the neuroprotection mediated by "anti-tau" activity of FKBP52 is linked to proteasomal degradation of hyperphosphorylated tau via enhanced trafficking or by increased aggregation of toxic tau into fibrillary tangles.

FKBP51, a member of the HSP90 chaperone complex, directly associates with tau and its overexpression significantly increases the levels of phospho- and total tau in cells. Contrary to FKBP52, FKBP51 enhances the tau-mediated MT polymerization and the PPIase activity of FKBP51 is crucial for its function in tau processing. The data by Dickey and coworkers suggest a model whereby binding of FKBP51-HSP90 complex to phosphorylated tau triggers its dephosphorylation and recycling to the microtubules thereby facilitating MT polymerization and stabilization (Jinwal et al., 2010). HSP90-FKBP51 binding also shields tau from CHIP (carboxy terminus of the HSC70-interacting protein) mediated ubiquitination and subsequent proteasomal degradation (Figure 3).

\subsection{FKBP38 anchors $\mathrm{Bcl}-2$ to the mitochondria and regulates apoptosis}

The C-terminal tail of the noncanonical FKBP, FKBP38, localizes the protein to the ER and mitochondrial membrane where it interacts with the anti-apoptotic proteins, Bcl-2 and Bcl- $\mathrm{x}_{\mathrm{L}}$ and regulates their functions. FKB38 is critical for the mitochondrial localization of Bcl-2 and $\mathrm{Bcl}-\mathrm{x}_{\mathrm{L} ;}$ expression of mitochondrial targeting defective FKBP38 mutants and RNAi mediated 


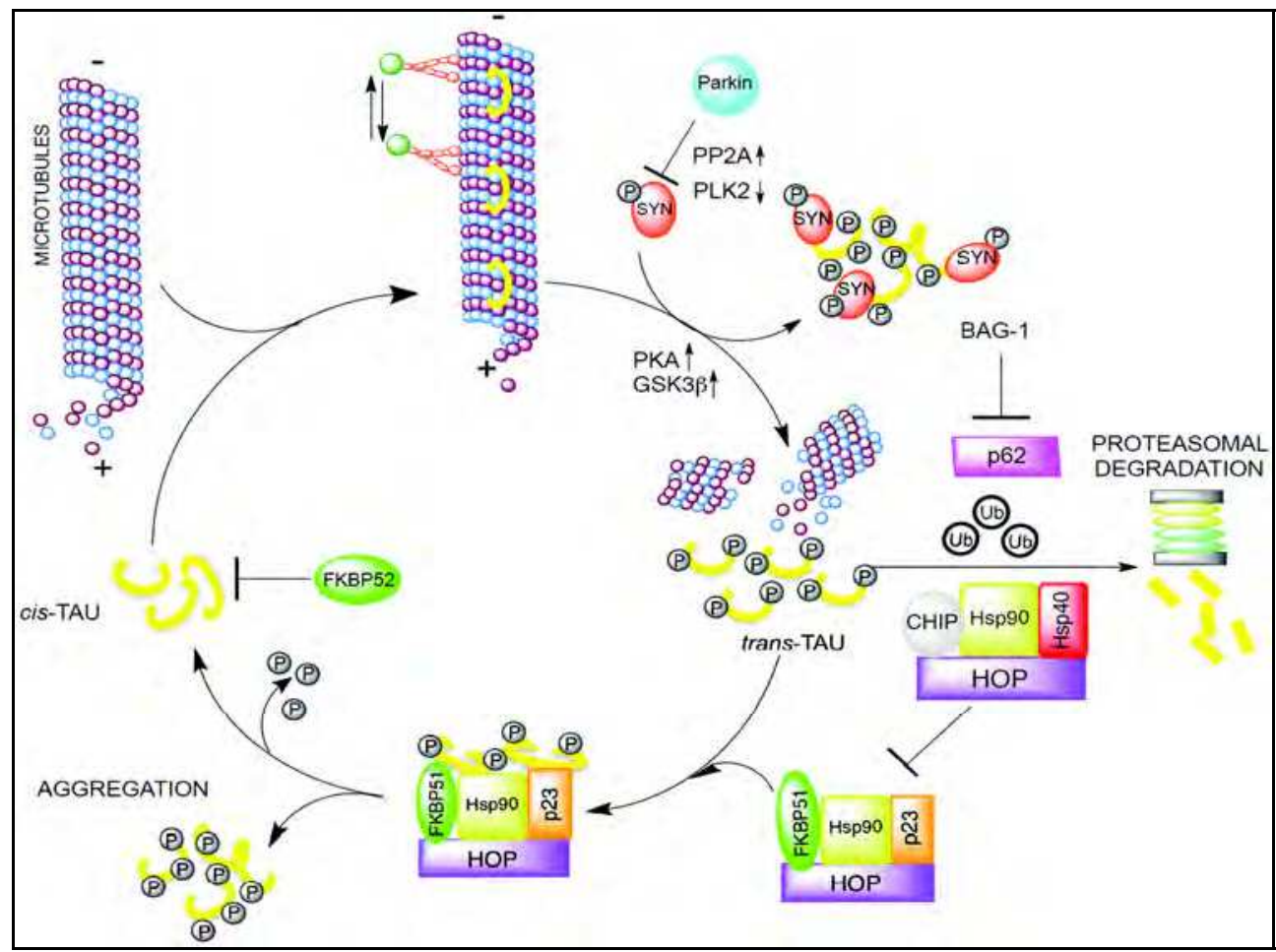

Fig. 3. FKBPs regulate microtubule stability by interacting with the microtubule-associated protein, tau. FKBP51 and FKBP52 have opposing effects on microtubule (MT) stability. FKBP52 exhibits "anti-tau" activity; by sequestering tau, it prevents its association with MT thereby destabilizing MTs. Together with the HSP90 complex, FKBP51 binds phosphorylated tau in its trans-conformation, isomerizes it to the cis-form and recycles it back to the MTs. Alternatively FKBP51 can accelerate aggregation of phospho-tau species. Phospho-tau can also be degraded by the cellular proteasomal system following CHIPmediated polyubiquitination. Binding of FKBP51 and CHIP to phosphorylated tau is mutually exclusive. By recruiting PKA/GSK3 $\beta$, $\alpha$-SYN promotes tau-phosphorylation within the MT-binding domain and its subsequent removal from MTs. Activity of $\alpha-S Y N$ is in turn regulated by the E3 ubiquitin ligase, Parkin. By inhibiting PLK2 kinase and enhancing PP2A phosphatase activity, Parkin decreases $\alpha$-SYN phosphorylation and interferes with its activity.

knockdown of FKBP38 causes the cellular redistribution of these proteins. Furthermore FKBP38-mediated mitochondrial targeting is responsible for the anti-apoptotic activity of FKBP38 (Shirane \& Nakayama, 2003; Kang et al., 2005). Nishimura and colleagues have shown FKBP38 is a bona fide substrate for the aspartyl protease, presenilin 1 and 2 (PS1/2) (Wang et al., 2005). Under physiological conditions PS1/2 forms macromolecular heteromeric complexes with FKBP38 and Bcl-2 and sequesters them in the ER/Golgi via a $\gamma$ secretase independent mechanism. Thus by inhibiting the FKBP38 mediated mitochondrial targeting of Bcl-2, PS1/2 antagonizes the anti-apoptotic effect of FKBP38. 
In neuroblastoma cells, FKBP38 exhibits $\mathrm{Ca} 2+/ \mathrm{CaM}$ stimulated PPIase activity and the FKBP38/Ca2+/CaM ternary complex binds Bcl-2. This binding is interrupted by GPI-1046, indicating that the active site of FKBP38 is involved in Bcl-2 interaction. HSP90 in the HSP90/Bcl-2/ $\mathrm{Ca}^{2+} / \mathrm{CaM}$ ternary complex has also been shown to inhibit the Bcl-2-FKBP38 interaction by blocking access to the enzyme active site (Edlich et al., 2007). GPI-1046 and RNAi-mediated depletion of FKBP38 activity was able to promote neuronal cell survival thus indicating that, in neuronal cells, FKBP38 has proapoptotic function. This observation contradicts earlier reports wherein it was shown that FKBP38 has anti-apoptotic effect (Shirane \& Nakayama, 2003; Kang et al., 2005). One plausible explanation for this discrepancy could be explained on the basis of the different cell lines, neuronal versus nonneuronal, used in these studies. The potent neuroprotective and neuroregenerative effects of low molecular weight FKBP38 inhibitors in neuroblastoma cells concur well with the proapoptotic role of FKBP38. Furthermore, the ability of the FKBP38 inhibitor - $N-\left(N^{\prime}, N^{\prime}-\right.$ dimethylcarboxamidomethyl)-cycloheximide to elicit neural stem proliferation and neuronal differentiation in a rat model of transient cerebral ischemia underscores the importance of FKBP38 in neuronal apoptosis (Edlich et al., 2006).

\section{Neuroimmunophilin ligands as therapeutics for PD}

Currently available drugs aimed at PD treatment do not have the capacity to inhibit PD progression but can only alleviate symptoms and/or delay neuronal atrophy by altering neurotransmitter metabolism. Neuroimmunophilin ligands are non-immunosuppressive and mediate the beneficial effects by multiple mechanisms that include inhibition of apoptosis, increased neurotrophic signaling and/or reducing oxidative stress by interfering with mitochondrial dysfunction (Tanaka \& Ogawa, 2004). Several groups have reported that NILs like FK506, GPI-1046 and V-10367 (Figure 4A) promote striatal dopaminergic innervations in MPTP- or 6-OHDA models of PD (Kitamura et al., 1994; Steiner et al., 1997; Costantini et al., 1998; Guo et al., 2001). Studies have also shown that GPI-1046 protects against the $p$-chloroamphetamine-induced destruction of central serotoninergic neurons and

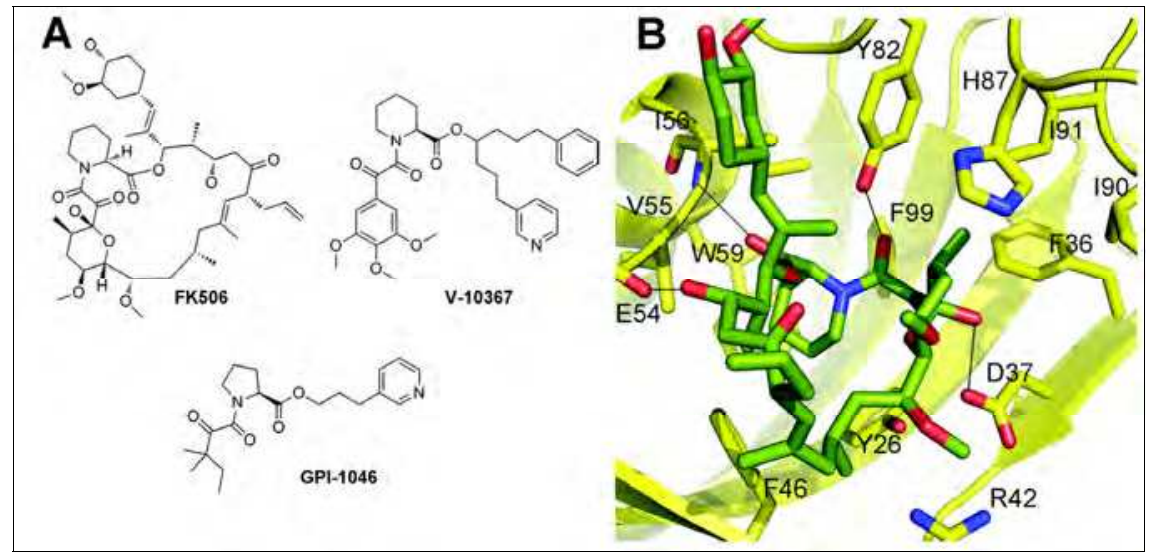

Fig. 4. (A) Structure of the neuroimmunophilin ligand FK506 and its nonimmunosuppressive derivatives, GPI-1046 and V-10367. (B) Binding of FK506 (green) into the active site pocket of FKBP12"(represented by cartoon model). 
senescence-related atrophy of medial septal cholinergic neurons (Sauer et al., 1999). Furthermore, rotational behavior and loss of corticostriatal long-term potentiation (LTP) in 6-OHDA treated rats was alleviated by GPI-1046. However, as similar efficacy was obtained with an analog that does not bind FKBP12 (V-13670), the importance of FKBP12 in mediating neurotrophic effects is debatable. Besides FK506 derivatives, CsA has been shown to protect against dopaminergic degeneration, promote regeneration of DA neurons and even suppress microglial cytotoxicity, as activated microglia has been known to generate free radicals (Banati et al., 1993).

The binding mode of FK506 and its non-immunosuppressive counterpart, GPI-1046 has been elucidated (Van Duyne et al., 1993; Sich et al., 2000). Minimal binding motif comprises of the central pipecolic acid ring, the $\alpha$-dicarbonyl amide linkage and pyranose ring. In the FK506-FKBP12 crystal structure (Van Duyne et al., 1993), the pipecoline ring sits in the cavity defined by Trp ${ }^{59}$ and $\mathrm{Tyr}^{26}$, Phe ${ }^{46}, \mathrm{Val}^{55}$, Ile ${ }^{56}, \mathrm{Phe}^{99}$ side chains, whereas the $\alpha$-dicarbonyl amide is hydrogen-bonded to $-\mathrm{COOH}$ and $-\mathrm{OH}$ group of $\mathrm{Asp}^{37}$ and $\mathrm{Tyr}^{82}$, respectively (Figure 4B). The hydrophobic pocket formed by $\mathrm{Phe}^{36}, \mathrm{Asp}^{37}, \mathrm{Tyr}^{82}, \mathrm{His}^{87}$, Ile ${ }^{90}$ and Ile ${ }^{91}$ buries the pyranose ring while the cyclohexyl ester chain is engaged in hydrophobic interactions within a shallow surface groove. SAR studies have shown that the $\alpha$-dicarbonyl amide functionality is indispensible for enzyme inhibition as replacement of either one or both carbonyl groups reduces potency. Similarly, pipecolic ring opening drastically increases the inhibition constant of the derivatives. GPI-1046 binds in a manner analogous to that of FK506 with the amide bond in trans configuration; the only exception being the replacement of pipecolyl moiety of FK506 by the prolyl ring of GPI-1046. Even though GPI1046 has fewer favorable protein-ligand interactions, its effect on protein dynamics is essentially same as FK506, that is, stabilize the conformation of solvent exposed residues that are important in protein-protein and protein-ligand interactions.

The therapeutic utility of NILs has been questioned by work from other groups (Harper et al., 1999; Parker et al., 2000; Bocquet et al., 2001) as many of the initial observations could not be replicated in identical systems. For instance, Harper and colleagues observed that GPI-1046 causes only a marginal increase in neurite outgrowth of chick dorsal root ganglia in culture under conditions where a very robust effect of nerve growth factor was seen. GPI-1046 did not restore tyrosine hydroxylase-positive fibers after 6-OHDA administration neither did it protect cultured dopaminergic neurons against MPTP induced toxicity. One possibility for the observed differences could be the levels of dopaminergic dysfunction. GPI-1046 provided neuroprotection in cases when the degradation was mild to moderate; it has no effect in severe cases when DA neuron levels deplete to $20 \%$ of that in normal controls. Other parameters like variability in culture conditions, differences in days in vitro prior to experimentation, can potentially account for the contrasting observations (Pong and Zaleska, 2003). Furthermore, neuroprotective effects of GPI-1046 could not be replicated in monkey model of MPTP toxicity (Emborg et al., 2001) suggesting species-specific differences with respect to GPI-1046 activity.

\section{Conclusion}

FKBPs have emerged as novel cellular target for treatment of Parkinson's disease and other neurological disorders. The extraordinary unmet need for therapeutic intervention in PD continues to drive the search for potential drug candidates. Non-immunosuppressive NILs with their small size, target specificity, bioavailability and stability provide excellent scaffolds for the development of new drugs. Although our present knowledge of the mode of action of NILs is still fragmentary, there is increasing evidence that the neurotrophic, 
neuroprotective and restorative potential of these compounds is mediated by signaling pathways that can have antagonistic or additive effect. For example, multiple pathways may crosstalk via common integral components such as c-Jun (Gold et al., 1999; Winter et al., 2000). So far, the nervous system has been found to be enriched in only five FKBPs - FKBP$12,-38,-51,-52$ and -65 . Much work needs to be done so as to identify unique neuroimmunophilins and their interacting partners, assess their cellular function as well as their response to injury. Furthermore, issues such as reproducibility of pre-clinical data, structure-activity relationship studies, drug evaluation in appropriate animal models, and implementation of proper clinical designs and endpoints needs immediate attention as such information will aid in the development of novel NILs with improved efficacies, target selectivity and potency. FKBPs and NILs seem to be a promising area for therapeutic intervention of PD and other neurodegenerative disorders.

\section{Acknowledgment}

The authors would like to acknowledge the support from Ministry of Health-National Medical Research Council, Singapore for funding (NMRC/1177/2008).

\section{References}

Auluck, P.K., Chan, H.Y., Trojanowski, J.Q., Lee, V.M. \& Bonini, N.M. (2002). Chaperone suppression of alpha-synuclein toxicity in a Drosophila model for Parkinson's disease. Science, Vol 295, pp 865-868.

Avramut, M. \& Achim, C.L. (2002). Immunophilins and their ligands: insights into survival and growth of human neurons. Physiol Behav, Vol 77, pp 463-468.

Banati, R.B., Gehrmann, J., Schubert, P. \& Kreutzberg, G.W. (1993). Cytotoxicity of microglia. Glia, Vol 7, pp 111-118.

Barik, S. (2006). Immunophilins: for the love of proteins. Cell Mol. Life Sci., Vol 63, pp 2889-2900.

Bocquet, A., Lorent, G., Fuks, B., Grimee, R., Talaga, P., Daliers, J. \& Klitgaard, H. (2001). Failure of GPI compounds to display neurotrophic activity in vitro and in vivo. Eur. J Pharmacol., Vol 415, pp 173-180.

Chambraud, B., Belabes, H., Fontaine-Lenoir, V., Fellous, A. \& Baulieu, E.E. (2007). The immunophilin FKBP52 specifically binds to tubulin and prevents microtubule formation. FASEB J., Vol 21, pp 2787-2797.

Chambraud, B., Sardin, E., Giustiniani, J., Dounane, O., Schumacher, M., Goedert, M. \& Baulieu, E.E. (2010). A role for FKBP52 in Tau protein function. Proc. Natl. Acad. Sci. USA, Vol 107, pp 2658-2663.

Charters, A.R., Kobayashi, M. \& Butcher, S.P. (1994). Immunochemical analysis of FK506 binding proteins in neuronal cell lines and rat brain. Biochem. Soc. Trans., Vol 22, pp $411 S$.

Coss, M.C., Stephens, R.M., Morrison, D.K., Winterstein, D., Smith, L.M. \& Simek, S.L. (1998). The immunophilin FKBP65 forms an association with the serine/threonine kinase c-Raf-1. Cell Growth Differ., Vol 9, pp 41-48.

Costantini, L.C., Chaturvedi, P., Armistead, D.M., McCaffrey, P.G., Deacon, T.W. \& Isacson, O. (1998). A novel immunophilin ligand: distinct branching effects on dopaminergic neurons in culture and neurotrophic actions after oral administration in an animal model of Parkinson's disease. Neurobiol. Dis Vol 5, pp 97-106.

Dawson, T.M., Steiner, J.P., Lyons, W.E., Fotuhi, M., Blue, M. \& Snyder, S.H. (1994). The immunophilins, FK506 binding protein and cyclophilin, are discretely localized in the brain: relationship to calcineurin. Neuroscience, Vol 62, pp 569-580. 
Dawson, T.M. (2006). Parkin and defective ubiquitination in Parkinson's disease. J. Neural Transm. Suppl, Vol 209-213.

Duka, T., Duka, V., Joyce, J.N. \& Sidhu, A. (2009). Alpha-Synuclein contributes to GSK3beta-catalyzed Tau phosphorylation in Parkinson's disease models. FASEB J, Vol 23, pp 2820-2830.

Edlich, F., Weiwad, M., Erdmann, F., Fanghanel, J., Jarczowski, F., Rahfeld, J.U. \& Fischer, G. (2005). Bcl-2 regulator FKBP38 is activated by Ca2+/calmodulin. EMBO J, Vol 24, pp 2688-2699.

Edlich, F., Weiwad, M., Wildemann, D., Jarczowski, F., Kilka, S., Moutty, M.C., Jahreis, G., Lucke, C., Schmidt, W., Striggow, F. \& Fischer, G. (2006). The specific FKBP38 inhibitor $\mathrm{N}-\left(\mathrm{N}^{\prime}, \mathrm{N}^{\prime}\right.$-dimethylcarboxamidomethyl)cycloheximide has potent neuroprotective and neurotrophic properties in brain ischemia. J Biol. Chem. Vol 281, pp 14961-14970.

Edlich, F., Erdmann, F., Jarczowski, F., Moutty, M.C., Weiwad, M. \& Fischer, G. (2007). The Bcl-2 regulator FKBP38-calmodulin-Ca2+ is inhibited by Hsp90. J Biol. Chem., Vol 282, pp 15341-15348.

Emborg, M.E., Shin, P., Roitberg, B., Sramek, J.G., Chu, Y., Stebbins, G.T., Hamilton, J.S., Suzdak, P.D., Steiner, J.P. \& Kordower, J.H. (2001). Systemic administration of the immunophilin ligand GPI 1046 in MPTP-treated monkeys. Exp. Neurol., Vol 168, pp 171-182.

Eriksen, J.L., Wszolek, Z. \& Petrucelli, L. (2005). Molecular pathogenesis of Parkinson disease. Arch. Neurol. Vol 62, pp 353-357.

Funauchi, M., Haruta, H. \& Tsumoto, T. (1994). Effects of an inhibitor for calcium/calmodulin-dependent protein phosphatase, calcineurin, on induction of long-term potentiation in rat visual cortex. Neurosci. Res., Vol 19, pp 269-278.

Fusco, D., Vargiolu, M., Vidone, M., Mariani, E., Pennisi, L.F., Bonora, E., Capellari, S., Dirnberger, D., Baumeister, R., Martinelli, P. \& Romeo, G. (2010). The RET51/FKBP52 complex and its involvement in Parkinson disease. Hum. Mol. Genet., Vol 19, pp 2804-2816.

Gerard, M., Debyser, Z., Desender, L., Kahle, P.J., Baert, J., Baekelandt, V. \& Engelborghs, Y. (2006). The aggregation of alpha-synuclein is stimulated by FK506 binding proteins as shown by fluorescence correlation spectroscopy. FASEB J., Vol 20, pp 524-526.

Gerard, M., Debyser, Z., Desender, L., Baert, J., Brandt, I., Baekelandt, V. \& Engelborghs, Y. (2008). FK506 binding protein 12 differentially accelerates fibril formation of wild type alpha-synuclein and its clinical mutants A30P or A53T. J Neurochem., Vol 106, pp 121-133.

Gerard, M., Deleersnijder, A., Daniels, V., Schreurs, S., Munck, S., Reumers, V., Pottel, H., Engelborghs, Y., Van den Haute, C., Taymans, J.M., Debyser, Z. \& Baekelandt, V. (2010). Inhibition of FK506 binding proteins reduces alpha-synuclein aggregation and Parkinson's disease-like pathology. J Neurosci., Vol 30, pp 2454-2463.

Gerlach, M., Riederer, P., Przuntek, H. \& Youdim, M.B. (1991). MPTP mechanisms of neurotoxicity and their implications for Parkinson's disease. Eur. J Pharmacol., Vol 208, pp 273-286.

Giasson, B.I., Forman, M.S., Higuchi, M., Golbe, L.I., Graves, C.L., Kotzbauer, P.T., Trojanowski, J.Q. \& Lee, V.M. (2003). Initiation and synergistic fibrillization of tau and alpha-synuclein. Science, Vol 300, pp 636-640.

Glass, C.K., Saijo, K., Winner, B., Marchetto, M.C. \& Gage, F.H. (2010). Mechanisms underlying inflammation in neurodegeneration. Cell, Vol 140, pp 918-934. 
Goedert, M. (2001). Alpha-synuclein and neurodegenerative diseases. Nat. Rev. Neurosci., Vol 2, pp 492-501.

Gold, B.G. (1997). FK506 and the role of immunophilins in nerve regeneration. Mol. Neurobiol., Vol 15, pp 285-306.

Gold, B.G. \& Nutt, J.G. (2002). Neuroimmunophilin ligands in the treatment of Parkinson's disease. Curr. Opin. Pharmacol., Vol 2, pp 82-86.

Gold, B.G., Densmore, V., Shou, W., Matzuk, M.M. \& Gordon, H.S. (1999). Immunophilin FK506-binding protein 52 (not FK506-binding protein 12) mediates the neurotrophic action of FK506. J Pharmacol. Exp. Ther., Vol 289, pp 1202-1210.

Guo, X., Dawson, V.L. \& Dawson, T.M. (2001). Neuroimmunophilin ligands exert neuroregeneration and neuroprotection in midbrain dopaminergic neurons. Eur. J Neurosci., Vol 13, pp 1683-1693.

Hamilton, G.S. \& Steiner, J.P. (1998). Immunophilins: beyond immunosuppression. J Med. Chem., Vol 41, pp 5119-5143.

Harper, S., Bilsland, J., Young, L., Bristow, L., Boyce, S., Mason, G., Rigby, M., Hewson, L., Smith, D., O'Donnell, R., O'Connor, D., Hill, R.G., Evans, D., Swain, C., Williams, B. \& Hefti, F. (1999). Analysis of the neurotrophic effects of GPI-1046 on neuron survival and regeneration in culture and in vivo. Neuroscience, Vol 88, pp 257-267.

Hodgkiss, J.P. \& Kelly, J.S. (1995). Only 'de novo' long-term depression (LTD) in the rat hippocampus in vitro is blocked by the same low concentration of FK506 that blocks LTD in the visual cortex. Brain Res., Vol 705, pp 241-246.

Hurst, R.S., Higdon, N.R., Lawson, J.A., Clark, M.A., Rutherford-Root, K.L., McDonald, W.G., Haas, J.V., McGrath, J.P. \& Meglasson, M.D. (2003). Dopamine receptor agonists differ in their actions on cardiac ion channels. Eur. J Pharmacol., Vol 482, pp 31-37.

Ischiropoulos, H. (2003). Oxidative modifications of alpha-synuclein. Ann. N. Y. Acad. Sci., Vol 991, pp 93-100.

Ishizawa, T., Mattila, P., Davies, P., Wang, D. \& Dickson, D.W. (2003). Colocalization of tau and alpha-synuclein epitopes in Lewy bodies. J Neuropathol. Exp. Neurol., Vol 62, pp 389-397.

Jensen, P.H., Hager, H., Nielsen, M.S., Hojrup, P., Gliemann, J. \& Jakes, R. (1999). Alphasynuclein binds to Tau and stimulates the protein kinase A-catalyzed tau phosphorylation of serine residues 262 and 356. J Biol. Chem., Vol 274, pp 25481-25489.

Jinwal, U.K., Koren, J., Borysov, S.I., Schmid, A.B., Abisambra, J.F., Blair, L.J., Johnson, A.G., Jones, J.R., Shults, C.L., O'Leary, J.C., Jin, Y., Buchner, J., Cox, M.B. \& Dickey, C.A. (2010). The Hsp90 cochaperone, FKBP51, increases Tau stability and polymerizes microtubules. J Neurosci., Vol 30, pp 591-599.

Kang, C.B., Feng, L., Chia, J. \& Yoon, H.S. (2005). Molecular characterization of FK-506 binding protein 38 and its potential regulatory role on the anti-apoptotic protein Bcl-2. Biochem. Biophys. Res. Commun., Vol 337, pp 30-38.

Khandelwal, P.J., Dumanis, S.B., Feng, L.R., Maguire-Zeiss, K., Rebeck, G., Lashuel, H.A. \& Moussa, C.E. (2010). Parkinson-related parkin reduces alpha-Synuclein phosphorylation in a gene transfer model. Mol. Neurodegener. Vol 5, pp 47.

Kitamura, Y., Itano, Y., Kubo, T. \& Nomura, Y. (1994). Suppressive effect of FK-506, a novel immunosuppressant, against MPTP-induced dopamine depletion in the striatum of young C57BL/ 6 mice. J Neuroimmunol., Vol 50, pp 221-224.

Kopito, R.R. (2000). Aggresomes, inclusion bodies and protein aggregation. Trends Cell Biol., Vol 10, pp 524-530.

Kragh, C.L., Lund, L.B., Febbraro, F., Hansen, H.D., Gai, W.P., El-Agnaf, O., Richter-Landsberg, C. \& Jensen, P.H. (2009). \{alpha\}-Synuclein Aggregation and Ser-129 Phosphorylationdependent Cell Death in Oligodendroglial Cells. J Biol. Chem., Vol 284, pp 10211-10222. 
Lyons, W.E., George, E.B., Dawson, T.M., Steiner, J.P. \& Snyder, S.H. (1994). Immunosuppressant FK506 promotes neurite outgrowth in cultures of PC12 cells and sensory ganglia. Proc. Natl. Acad. Sci. USA, Vol 91, pp 3191-3195.

Maki, N., Sekiguchi, F., Nishimaki, J., Miwa, K., Hayano, T., Takahashi, N. \& Suzuki, M. (1990). Complementary DNA encoding the human T-cell FK506-binding protein, a peptidylprolyl cis-trans isomerase distinct from cyclophilin. Proc. Natl. Acad. Sci. USA, Vol 87, pp 5440-5443.

Mandel, S., Grunblatt, E., Riederer, P., Amariglio, N., Jacob-Hirsch, J., Rechavi, G. \& Youdim, M.B. (2005). Gene expression profiling of sporadic Parkinson's disease substantia nigra pars compacta reveals impairment of ubiquitin-proteasome subunits, SKP1A, aldehyde dehydrogenase, and chaperone HSC-70. Ann. N. Y. Acad. Sci., Vol 1053, pp 356-375.

McGeer, P.L., Itagaki, S., Boyes, B.E. \& McGeer, E.G. (1988). Reactive microglia are positive for HLA-DR in the substantia nigra of Parkinson's and Alzheimer's disease brains. Neurology, Vol 38, pp 1285-1291.

Meiri, K.F., Bickerstaff, L.E. \& Schwob, J.E. (1991). Monoclonal antibodies show that kinase C phosphorylation of GAP-43 during axonogenesis is both spatially and temporally restricted in vivo. J Cell Biol., Vol 112, pp 991-1005.

Meuvis, J., Gerard, M., Desender, L., Baekelandt, V. \& Engelborghs, Y. (2010). The conformation and the aggregation kinetics of alpha-synuclein depend on the proline residues in its C-terminal region. Biochemistry, Vol 49, pp 9345-9352.

Muchowski, P.J. (2002). Protein misfolding, amyloid formation, and neurodegeneration: a critical role for molecular chaperones? Neuron, Vol 35, pp 9-12.

Muntane, G., Dalfo, E., Martinez, A. \& Ferrer, I. (2008). Phosphorylation of tau and alphasynuclein in synaptic-enriched fractions of the frontal cortex in Alzheimer's disease and in Parkinson's disease and related alpha-synucleinopathies. Neuroscience, Vol 152, pp 913-923.

Nagatsu, T. \& Sawada, M. (2005) Inflammatory process in Parkinson's disease: role for cytokines. Curr. Pharm. Des., Vol 11, pp 999-1016.

Nichols, R.A., Suplick, G.R. \& Brown, J.M. (1994). Calcineurin-mediated protein dephosphorylation in brain nerve terminals regulates the release of glutamate. $J$ Biol. Chem., Vol 269, pp 23817-23823.

Nilsson, A., Skold, K., Sjogren, B., Svensson, M., Pierson, J., Zhang, X., Caprioli, R.M., Buijs, J., Persson, B., Svenningsson, P \& Andren, P.E. (2007). Increased striatal mRNA and protein levels of the immunophilin FKBP-12 in experimental Parkinson's disease and identification of FKBP-12-binding proteins. J Proteome Res., Vol 6, pp 3952-3961.

Parker, E.M., Monopoli, A., Ongini, E., Lozza, G. \& Babij. C.M. (2000). Rapamycin, but not FK506 and GPI-1046, increases neurite outgrowth in PC12 cells by inhibiting cell cycle progression. Neuropharmacology, Vol 39, pp 1913-1919.

Pong, K. \& Zaleska, M.M. (2003). Therapeutic implications for immunophilin ligands in the treatment of neurodegenerative diseases. Curr. Drug Targets CNS Neurol. Disord ., Vol 2, pp 349-356.

Poon, H.F., Shepherd, H.M., Reed, T.T., Calabrese, V., Stella, A.M., Pennisi, G., Cai, J., Pierce, W.M., Klein, J.B. \& Butterfield, D.A. (2006). Proteomics analysis provides insight into caloric restriction mediated oxidation and expression of brain proteins associated with age-related impaired cellular processes: Mitochondrial dysfunction, glutamate dysregulation and impaired protein synthesis. Neurobiol. Aging., Vol 27, pp 1020-1034.

Sauer, H., Francis, J.M., Jiang, H., Hamilton, G.S. \& Steiner, J.P. (1999). Systemic treatment with GPI 1046 improves spatial memory and reverses cholinergic 
neuron atrophy in the medial septal nucleus of aged mice. Brain Res., Vol 842, pp 109-118.

Sharkey, J. \& Butcher, S.P. (1994). Immunophilins mediate the neuroprotective effects of FK506 in focal cerebral ischaemia. Nature, Vol 371, pp 336-339.

Sharma, V.K., Li, B., Khanna, A., Sehajpal, P.K. \& Suthanthiran, M. (1994). Which way for drug-mediated immunosuppression? Curr. Opin. Immunol., Vol 6, pp 784-790.

Shirane, M. \& Nakayama, K.I. (2003). Inherent calcineurin inhibitor FKBP38 targets Bcl-2 to mitochondria and inhibits apoptosis. Nat. Cell Biol., Vol 5, pp 28-37.

Sich, C., Improta, S., Cowley, D.J., Guenet, C., Merly, J.P., Teufel, M \& Saudek, V. (2000). Solution structure of a neurotrophic ligand bound to FKBP12 and its effects on protein dynamics. Eur. J Biochem., Vol 267, pp 5342-5355.

Snyder, S.H. (1992). Nitric Oxide: first in a new class of neurotransmitters. Science, Vol 257, pp 494-496.

Snyder, S.H., Lai, M.M. \& Burnett, P.E. (1998). Immunophilins in the nervous system. Neuron, Vol 21, pp 283-294.

Steiner, J.P., Dawson, T.M., Fotuhi, M., Glatt, C.E., Snowman, A.M., Cohen, N \& Snyder, S.H. (1992). High brain densities of the immunophilin FKBP colocalized with calcineurin. Nature, Vol 358, pp 584-587.

Steiner, J.P., Dawson, T.M., Fotuhi, M \& Snyder, S.H. (1996). Immunophilin regulation of neurotransmitter release. Mol. Med., Vol 2, pp 325-333.

Steiner, J.P., Hamilton, G.S., Ross, D.T., Valentine, H.L., Guo, H., Connolly, M.A., Liang, S., Ramsey, C., Li, J.H., Huang, W., Howorth, P., Soni, R., Fuller, M., Sauer, H., Nowotnik, A.C. \& Suzdak, P.D. (1997). Neurotrophic immunophilin ligands stimulate structural and functional recovery in neurodegenerative animal models. Proc. Natl. Acad. Sci. USA, Vol 94, pp 2019-2024.

Tanaka, K. \& Ogawa, N. (2004). Possibility of non-immunosuppressive immunophilin ligands as potential therapeutic agents for Parkinson's disease. Curr. Pharm. Des., Vol 10, pp 669-677.

Tong, G., Shepherd, D. \& Jahr, C.E. (1995). Synaptic desensitization of NMDA receptors by calcineurin. Science, Vol 267, pp 1510-1512.

Tong, Y. \& Shen, J. (2009). Alpha-synuclein and LRRK2: partners in crime. Neuron, Vol 64, pp 771-773.

Van Duyne, G.D., Standaert, R.F., Karplus, P.A., Schreiber, S.L. \& Clardy, J. (1993). Atomic structures of the human immunophilin FKBP-12 complexes with FK506 and rapamycin. J Mol. Biol., Vol 229, pp 105-124.

Walker, V.E., Atanasiu, R., Lam, H. \& Shrier, A. (2007). Co-chaperone FKBP38 promotes HERG trafficking. J Biol. Chem., Vol 282, pp 23509-23516.

Wang, H.Q., Nakaya, Y., Du, Z., Yamane, T., Shirane, M., Kudo, T., Takeda, M., Takebayashi, K., Noda, Y., Nakayama, K.I. \& Nishimura, M. (2005). Interaction of presenilins with FKBP38 promotes apoptosis by reducing mitochondrial Bcl-2. Hum. Mol. Genet., Vol 14, pp 1889-1902.

Wang, X., Venable, J., LaPointe, P., Hutt, D.M., Koulov, A.V., Coppinger, J., Gurkan, C., Kellner, W., Matteson, J., Plutner, H., Riordan, J.R., Kelly, J.W., Yates, JR. \& Balch, W.E. (2006). Hsp90 cochaperone Aha1 downregulation rescues misfolding of CFTR in cystic fibrosis. Cell, Vol 127, pp 803-815.

Winter, C., Schenkel, J., Burger, E., Eickmeier, C., Zimmermann, M \& Herdegen, T. (2000). The immunophilin ligand FK506, but not GPI-1046, protects against neuronal death and inhibits c-Jun expression in the substantia nigra pars compacta following transection of the rat medial forebrain bundle. Neuroscience, Vol 95, pp 753-762. 


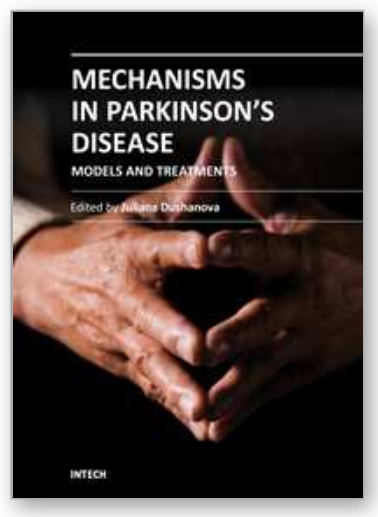

\author{
Mechanisms in Parkinson's Disease - Models and Treatments \\ Edited by Dr. Juliana Dushanova
}

ISBN 978-953-307-876-2

Hard cover, 582 pages

Publisher InTech

Published online 08, February, 2012

Published in print edition February, 2012

Parkinson's disease (PD) results primarily from the death of dopaminergic neurons in the substantia nigra. Current PD medications treat symptoms; none halt or retard dopaminergic neuron degeneration. The main obstacle to developing neuroprotective therapies is a limited understanding of the key molecular mechanisms that provoke neurodegeneration. The discovery of PD genes has led to the hypothesis that misfolding of proteins and dysfunction of the ubiquitin-proteasome pathway are pivotal to PD pathogenesis. Previously implicated culprits in PD neurodegeneration, mitochondrial dysfunction, and oxidative stress may also act in part by causing the accumulation of misfolded proteins, in addition to producing other deleterious events in dopaminergic neurons. Neurotoxin-based models have been important in elucidating the molecular cascade of cell death in dopaminergic neurons. PD models based on the manipulation of PD genes should prove valuable in elucidating important aspects of the disease, such as selective vulnerability of substantia nigra dopaminergic neurons to the degenerative process.

\title{
How to reference
}

In order to correctly reference this scholarly work, feel free to copy and paste the following:

Souvik Chattopadhaya, Amaravadhi Harikishore and Ho Sup Yoon (2012). Role of FKBPs in Parkinson's Disease, Mechanisms in Parkinson's Disease - Models and Treatments, Dr. Juliana Dushanova (Ed.), ISBN: 978-953-307-876-2, InTech, Available from: http://www.intechopen.com/books/mechanisms-in-parkinson-sdisease-models-and-treatments/role-of-fkbps-in-parkinson-s-disease

\section{INTECH}

open science | open minds

\section{InTech Europe}

University Campus STeP Ri

Slavka Krautzeka 83/A

51000 Rijeka, Croatia

Phone: +385 (51) 770447

Fax: +385 (51) 686166

www.intechopen.com

\section{InTech China}

Unit 405, Office Block, Hotel Equatorial Shanghai

No.65, Yan An Road (West), Shanghai, 200040, China

中国上海市延安西路65号上海国际贵都大饭店办公楼 405 单元

Phone: +86-21-62489820

Fax: $+86-21-62489821$ 
(C) 2012 The Author(s). Licensee IntechOpen. This is an open access article distributed under the terms of the Creative Commons Attribution 3.0 License, which permits unrestricted use, distribution, and reproduction in any medium, provided the original work is properly cited. 